\title{
Strain history of ice shells of the Galilean satellites from radar detection of crystal orientation fabric
}

\author{
Amy C. Barr ${ }^{1}$ and David E. Stillman ${ }^{1}$ \\ Received 29 December 2010; revised 4 February 2011; accepted 11 February 2011; published 24 March 2011.
}

[1] Orbital radar sounding has been suggested as a means of determining the subsurface thermal and physical structure of the outer ice I shells of the Galilean satellites. At radar frequencies, the dielectric permittivity of singleand polycrystalline water ice I is anisotropic. Crystal orientation fabric (COF), which is indicative of strain history, can be unambiguously detected by comparing the received power of dual co-polarization (linear polarization parallel and perpendicular to the orbit) radar data. Regions with crystal orientations dictated by the local strain field ("fabric") form in terrestrial ice masses where accumulated strain and temperature are high, similar to conditions expected in a convecting outer ice I shell on Europa, Ganymede, or Callisto. We use simulations of solid-state ice shell convection to show that crystal orientation fabric can form in the warm convecting sublayer of the ice shells for plausible grain sizes. Changes in received power from parallel and perpendicular polarizations in the ice shells due to fabric could be detected if multi-polarization data is collected. With proper instrument design, radar sounding could be used to shed light on the strain history of the satellites' ice shells in addition to their present day internal structures. Citation: Barr, A. C., and D. E. Stillman (2011), Strain history of ice shells of the Galilean satellites from radar detection of crystal orientation fabric, Geophys. Res. Lett., 38, L06203, doi:10.1029/2010GL046616.

\section{Introduction}

[2] The next mission to the Jovian satellites will likely carry an ice-penetrating radar to study the subsurface physical and thermal structure of the floating outer ice I shells of Europa and Ganymede [Blankenship et al., 2009]. Because radar attenuation in ice is caused primarily by temperature and salinity [Evans, 1965; Glen and Paren, 1975], studies of the ice shells' internal temperature and salt distributions [Chyba et al., 1998; Moore, 2000] have been key for constraining sounder design parameters.

[3] The orientation of the ice crystal lattice also affects the permittivity of ice. Deformation in polycrystalline ice can change the size and orientation of the crystal lattice of individual grains [Alley, 1988]. When a stress is applied, grains deform by basal slip and their crystallographic $c$ axes rotate toward the axis of compression [Alley, 1988]. Large strains at high temperatures create regions wherein the lattices of adjacent crystals are co-aligned (crystal orientation fabric or COF). Ice core samples suggest that fabric forms

\footnotetext{
${ }^{1}$ Department of Space Studies, Southwest Research Institute, Boulder, Colorado, USA.

Copyright 2011 by the American Geophysical Union. 0094-8276/11/2010GL046616
}

where the total accumulated strain, $\varepsilon>0.5$ [Alley, 1988; Budd and Jacka, 1989]. Seasonally average temperatures in most ice cores are $T>238 \mathrm{~K}\left(-35^{\circ} \mathrm{C}\right)$. These conditions are similar to conditions within the warm regions of a convecting ice shell on the Galilean satellites [Tobie et al., 2003].

[4] On Earth, COF detected by seismic [Blankenship, 1989] and radar sounding [Siegert and Kwok, 2000; Siegert and Fujita, 2001; Matsuoka et al., 2003; Dall, 2009, 2010] has been used to infer the strain history of ice sheets. For single-crystal and polycrystalline ice with well-developed COF ( $\varepsilon \gg 1$ at high temperature), the difference between the real part of the dielectric permittivity $\epsilon$, in a direction parallel to $\left(\epsilon_{\|}\right)$and perpendicular to $\left(\epsilon_{\perp}\right)$ the $c$-axis is about $1 \%$ [Johari and Charette, 1975; Fujita et al., 1993], which causes radar reflections at abrupt contrasts in $\mathrm{COF}$, anisotropic reflectivity, and birefringence [Matsuoka et al., 2003]. Reflections due to changes in COF can be as large as $\sim-50 \mathrm{~dB}$ [Fujita et al., 1993], within the expected $-60 \mathrm{~dB}$ dynamic range for a proposed radar sounder for Europa [Blankenship et al., 2009], but these reflections cannot be separated from reflections caused by changes in conductivity or density. Anisotropic reflectivity and birefringence vary periodically with polarization angle [Hargreaves, 1977; Matsuoka et al., 2003]. Zones of anisotropic reflectivity and birefringence have been detected by comparing the received power of dual co-polarization (i.e., linear polarization parallel and perpendicular to the ground track) ice-penetrating radar data [Matsuoka et al., 2003; Dall, 2009, 2010]. Polarizationdependent reflectivity provides an unambiguous means of detecting COF and could be used to test hypotheses about ice shell heat transfer and modes of resurfacing.

[5] Here, we simulate solid-state convection in the outer ice I shells of Europa, Ganymede, and Callisto to determine whether the strain and temperature conditions are conducive to COF formation. A composite rheology [van den Berg et al., 1993; McNamara et al., 2001; Freeman et al., 2006] for water ice I based on laboratory experiments and microphysical models of the relevant deformation processes [Goldsby and Kohlstedt, 2001] is used. COF forms in locations where convective strain is accommodated principally by anisotropic creep mechanisms dislocation and grain size-sensitive creep [Alley, 1988; Goldsby and Kohlstedt, 2002; Karato, 2008]. We show that COF can form in the warm convecting sublayer of an ice I shell for $d>1 \mathrm{~mm}$. If the ice shells have a smaller uniform grain size, fabric forms only in convective downwellings. We also discuss implications for radar sounder design and the formation of COF beneath surface features such as Europa's bands and Ganymede's grooved terrain.

\section{The Model}

[6] Laboratory experiments suggest that the total strain rate $(\dot{\varepsilon})$ in ice is equal to the sum of strain rates from 
dislocation creep, grain size-sensitive creep (which occurs by grain boundary sliding and basal slip), and diffusion creep [Goldsby and Kohlstedt, 2001],

$$
\dot{\varepsilon}=\dot{\varepsilon}_{d i s l}+\left(\dot{\varepsilon}_{g b s}^{-1}+\dot{\varepsilon}_{b s}^{-1}\right)^{-1}+\dot{\varepsilon}_{d i f f},
$$

where the strain rate from each deformation mechanism is related to temperature $(T)$, stress $(\sigma)$, and grain size $(d)$,

$$
\eta_{i}=\frac{d^{p_{i}}}{2 A_{i}} \sigma^{1-n_{i}} \exp \left(\frac{Q_{i}^{*}}{R_{G} T}\right),
$$

with $\eta=\sigma / 2 \dot{\varepsilon}$, grain size exponent (for rheology $i$ ) $p_{i}$, stress exponent $n_{i}$, pre-exponential constant $A_{i}$, gas constant $R_{G}=$ $8.314 \mathrm{~J} \mathrm{~mol}^{-1} \mathrm{~K}^{-1}$ and activation energy $Q_{i}^{*}$. For dislocation creep, $A_{\text {disl }}=4.0 \times 10^{-19} \mathrm{~Pa}^{-4} \mathrm{~s}^{-1}, n_{\text {disl }}=4, p_{\text {disl }}=0$, and $Q_{\text {disl }}^{*}=60 \mathrm{~kJ} \mathrm{~mol}^{-1}$; for grain boundary sliding, $A_{g b s}=$ $6.2 \times 10^{-14} \mathrm{~m}^{1.4} \mathrm{~Pa}^{-1.8} \mathrm{~s}^{-1}, n_{g b s}=1.8, p_{g b s}=1.4, Q_{g b s}^{*}=$ $49 \mathrm{~kJ} \mathrm{~mol}^{-1}$; and for basal slip, $A_{b s}=2.2 \times 10^{-7} \mathrm{~Pa}^{-2.4} \mathrm{~s}^{-1}$, $n_{b s}=2.4$, and $p_{b s}=0$, and $Q_{b s}^{*}=60 \mathrm{~kJ} \mathrm{~mol}^{-1}$. The viscosity for diffusion creep is [Goodman et al., 1981; Barr and Showman, 2009],

$$
\eta_{\text {diff }}=\frac{1}{2} \frac{3 R_{G} T_{b} d^{2}}{42 V_{m} D_{o, v}} \exp \left(\frac{Q_{v}^{*}}{R_{G} T}\right),
$$

with molar volume $V_{m}=1.97 \times 10^{-5} \mathrm{~m}^{-3}$, volume diffusion coefficient $D_{o, v}=9.10 \times 10^{-4} \mathrm{~m}^{2} \mathrm{~s}^{-1}$, activation energy $Q_{v}^{*}=59.4 \mathrm{~kJ} \mathrm{~mol}^{-1}$, and basal temperature of the shell $T_{b}$. A composite rheology [van den Berg et al., 1993; McNamara et al., 2001; Freeman et al., 2006],

$$
\frac{1}{\eta_{t o t}}=\frac{1}{\eta_{\text {disl }}}+\left[\eta_{G B S}+\eta_{B S}\right]^{-1}+\frac{1}{\eta_{\text {diff }}},
$$

is used to account for contributions to the ice viscosity from each deformation mechanism.

[7] The same processes that drive $c$-axis orientation can form new grains by recrystallization [Alley, 1988], which can be tens to hundreds of $\mathrm{mm}$ in the Galilean satellites [Barr and McKinnon, 2007]. However, in terrestrial ice sheets with $\dot{\varepsilon}$ and $T$ similar to a convecting ice shell, $c$-axis rotation is thought to occur without concomitant recrystallization [Alley, 1988]. Accordingly, we assume that the ice shell has a uniform grain size $0.1 \mathrm{~mm}<d<30 \mathrm{~mm}$ kept constant by grain boundary pinning from small silicate particles [Kirk and Stevenson, 1987; Barr and McKinnon, 2007].

[8] The finite-element model CITCOM [Moresi and Solomatov, 1995] is used to simulate convection for the range of $d$ and ice shell thickness, $D$, appropriate for the satellites in a $1 \times 12$-dimensional Cartesian box with $64 \times$ 64 elements. Three-dimensional Cartesian calculations in a $4 \times 4 \times 1$ box with $97 \times 97 \times 33$ elements are performed to study the pattern of COF-forming regions.

[9] The surface is kept at a constant $T_{s}=110 \mathrm{~K}$, appropriate for the mid-latitudes of Europa, Ganymede, and Callisto [Ojakangas and Stevenson, 1989; Herrick and Stevenson, 1990] and the bottom is heated to maintain $T_{b}=260 \mathrm{~K}$. Free-slip boundary conditions are used on the sides of the domain. The Rayleigh number is,

$$
R a_{o}=\frac{\rho g \alpha \Delta T D^{3}}{\kappa \eta_{o}}
$$

where $\eta_{o}=\eta_{\text {diff }}\left(T=T_{b}\right.$ ) (see equation (3)), $\Delta T=T_{b}-T_{s}$, coefficient of thermal expansion $\alpha=10^{-4} \mathrm{~K}^{-1}$, ice density $\rho=920 \mathrm{~kg} \mathrm{~m}^{-3}$, thermal diffusivity $\kappa=10^{-6} \mathrm{~m}^{2} \mathrm{~s}^{-1}$, and gravity $g=1.3 \mathrm{~m} \mathrm{~s}^{-2}$, an average value roughly appropriate for all three satellites.

[10] For numerical tractability, we use a rheology of form [Solomatov and Moresi, 2000],

$$
\eta_{i}=\frac{1}{\beta_{i}} \sigma^{\prime 1-n_{i}} \exp \left(E_{i}\left(1-T^{\prime}\right)\right),
$$

for each term in the composite flow law, where $E_{i}=Q_{i}^{*} \Delta T /$ $\left(R_{G} T_{b}^{2}\right)$, and $\beta_{i}$ describes the contribution of each component rheology [van den Berg et al., 1993],

$$
\beta_{i}=\left(2 \eta_{o}\right)^{n_{i}} \varepsilon_{o}^{n_{i}-1} A_{i} d^{-p_{i}} \exp \left(\frac{-Q_{i}^{*}}{R_{G} T_{b}}\right) .
$$

Sample calculations performed with an Arrhenius version of the temperature dependence (equation (2)) give similar temperature and strain fields in the warm region of the ice shell. In the composite flow law, the non-dimensional stress $\sigma^{\prime}=\sigma / \sigma_{o}, \sigma_{o}=2 \eta_{o} \dot{\varepsilon}_{o}$, the reference strain rate $\dot{\varepsilon}_{o}=\kappa / D^{2}$, and $T^{\prime}=\left(T-T_{s}\right) /(\Delta T)$. Because the $\beta$ factors depend on $D$ and $d$, these properties must be specified a priori.

[11] We estimate strain in the ice shell as $\varepsilon \sim \dot{\varepsilon}\left(D^{2} / \kappa\right)$, by assuming that strain accumulates over at least one thermal diffusion time. Equation (6) underestimates the temperature dependence in the viscosity, so ice in the stagnant lid is unphysically soft and strains there should be interpreted as upper limits. COF forms where the ratio of strain due to dislocation + GSS creep to diffusion creep [McNamara et al., 2001],

$$
R=\frac{\varepsilon_{\text {disl }}+\varepsilon_{G S S}}{\varepsilon_{\text {diff }}},
$$

is greater than 1 (i.e., anisotropic strain dominates isotropic strain). Because little is known about COF formation at low temperatures, we also require $T>240 \mathrm{~K}$.

\section{Results}

[12] Figure 1 shows the values of $D$ and $d$ explored in the 2-dimensional Cartesian calculations. Figure 2 shows values of $R$ in a convecting ice shell for several different uniform grain sizes. Regardless of grain size, $R>1$ in the stagnant lid, but temperatures there are too low to form COF. Beneath the stagnant lid, $T>240 \mathrm{~K}, R>1$ and the total strain $\gg 1$ in the center of the single convective roll if $d>1 \mathrm{~mm}$, a plausible grain size for the Galilean satellites' ice shells [Kirk and Stevenson, 1987; Barr and McKinnon, 2007]. Because radar attenuation in pure ice is temperature-dependent (attenuation increases by a factor of 10 between 248 and $272 \mathrm{~K}$ [Johari and Charette, 1975]), $\mathrm{COF}$ and interfaces between regions with different fabric orientations will be detected in cold downwellings and within the stagnant lid. If the stagnant lid is thin (i.e., the 

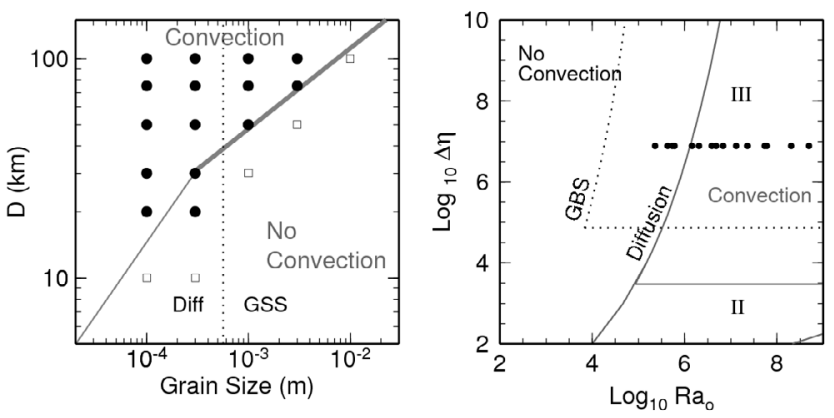

Figure 1. (left) Values of grain size and ice shell thickness explored in 2-dimensional simulations. Gray lines show the locations of the critical ice shell thickness for convection from Solomatov and Barr [2007], circles indicate simulations where convection occurred, and squares show where convection did not occur. Dotted line indicates boundary between $d$ values where GSS creep, rather than volume diffusion accommodates strain in the convective sublayer. (right) Parameter space in $R a_{o}-\Delta \eta$ space $\left(\Delta \eta \equiv\left(\eta_{\text {tot }}^{\prime}\left(T^{\prime}=0\right.\right.\right.$, $\left.\left.\left.\sigma^{\prime}=1\right)\right) /\left(\eta_{t o t}^{\prime}\left(T^{\prime}=1, \sigma^{\prime}=1\right)\right)\right)$. Gray lines indicate boundaries between convection/no convection and the stagnant lid (III) and sluggish lid (II) regimes $(\Delta \eta>\exp (4(n+1))$ where $n=1$ for volume diffusion) [Solomatov, 1995]. Dotted line shows regime boundaries for GBS $(n=1.8)$.

ice shell is thick and has a small grain size, a $-50 \mathrm{~dB}$ reflection would be detectable just above the stagnant lid. If the lid is thick (i.e., low $R a_{o}$ and large $d$ ), only strong reflections, $-10 \mathrm{~dB}$, could be detected at the base of the stagnant lid. In a salty ice shell (ice saturated with $\mathrm{Cl}^{-}$ [Grimm et al., 2008]), the search for COF would be confined to old patterns preserved in the cold stagnant lid.

[13] Figure 3 illustrates the temperature and $R$ values in a 30 kilometer-thick ice shell with $d=0.3 \mathrm{~mm}$. Values of $R$ are highest in downwellings and lowest in the upwellings. A horizontal slice through the shell at the location where the average $T=240 \mathrm{~K}$ shows that the diameter of regions with $R>1$ is about $25 \mathrm{~km}$, similar to the thickness of the ice shell. An instrument that could spatially resolve convective upwellings would thus be able to detect fabric formed in downwellings.

\section{Discussion}

[14] Detection of crystal orientation fabric by radar sounding has provided valuable insights into the strain history of terrestrial ice sheets. Our simulations of solidstate convection show that COF can form and be detected in convective downwellings in an ice shell with uniform grain sizes $d>0.3 \mathrm{~mm}$. For larger grain sizes $(d>1 \mathrm{~mm})$, COFforming regions encompass the entire ice shell. If the proposed Europa radar sounder acquires single-polarization data, only the strongest COF reflections will be detectable, but could be indistinguishable from reflections caused by changes in density, conductivity, and/or dust concentration. Multi-polarization radar could unambiguously detect COF and would require using two transmitting/receiving antennae perpendicular to each other, or flying two overlapping ground tracks with the spacecraft's radar antenna rotated $90^{\circ}$ in yaw on the second pass.
[15] A quantitative model of $c$-axis orientation and temperature based on the convective flow field should be meshed with a description of the locations of soluble/ insoluble impurities to fully address the dielectric properties of a convecting ice shell. Such a model could be used to predict the radar returns from a realistic ice shell including polarization effects. In addition, we have used a relatively simple rheological and convective model of the satellites' ice shells. The viscosity of ice with well-developed fabric is strongly anisotropic [Cuffey and Paterson, 2010], which could lead to changes in the convection pattern and predicted strain rates in the convecting sublayer. The viscosity of ice also depends on grain size, which in turn depends on impurity content, impurity solubility, and the relative importance of dynamic recrystallization, which can occur in tandem with COF formation [e.g., Durand et al., 2006; Barr and McKinnon, 2007; Cuffey and Paterson, 2010]. These effects should be included in future modeling efforts.

[16] Europa's ice shell is likely heated from within by tidal dissipation [Ojakangas and Stevenson, 1989], which can modify the convection pattern. The processes that convert tidal deformation to heat are poorly constrained [Barr and Showman, 2009], but a consensus is emerging that convective upwellings and shallow faults may be sites of intense heating [Nimmo and Gaidos, 2002; Mitri and Showman, 2008; Han and Showman, 2010]. Future work should address how tidal heating may affect the sites of $\mathrm{COF}$ formation within Europa's ice shell.

[17] Geological activity on Ganymede may have occurred only for a brief period [Pappalardo et al., 2004], and Europa's activity may be short-lived or even cyclical

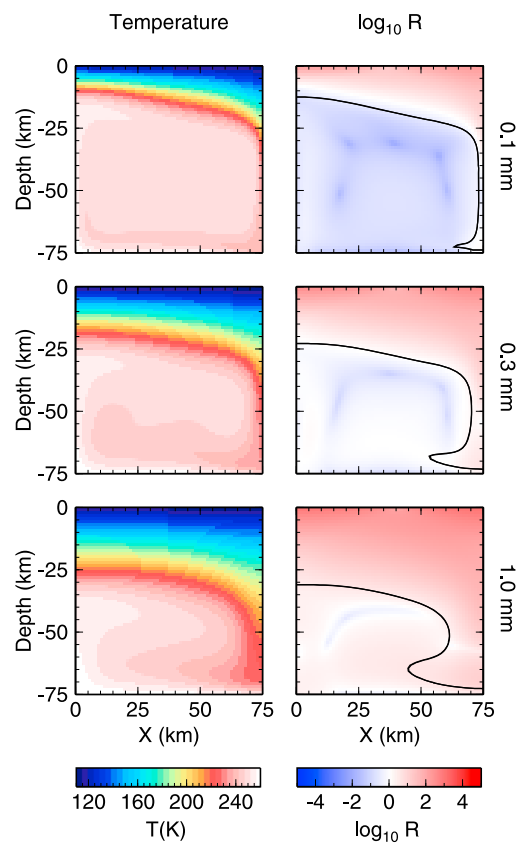

Figure 2. (left) Temperature and (right) $R$ for an ice shell $D=75 \mathrm{~km}$ thick, with a uniform grain size (top) $d=$ $0.1 \mathrm{~mm}\left(R a_{o}=2 \times 10^{8}\right)$, (middle) $d=0.3 \mathrm{~mm}\left(R a_{o}=2 \times\right.$ $\left.10^{7}\right)$, and (bottom) $d=1.0 \mathrm{~mm}\left(R a_{o}=2 \times 10^{6}\right)$. Black line shows the location of the $T=240 \mathrm{~K}$ isotherm. For $T>$ $240 \mathrm{~K}, \mathrm{COF}$ will form if $R>1$ (red values). Total accumulated strain $\varepsilon \gg 1$ everywhere $T>240 \mathrm{~K}$. 


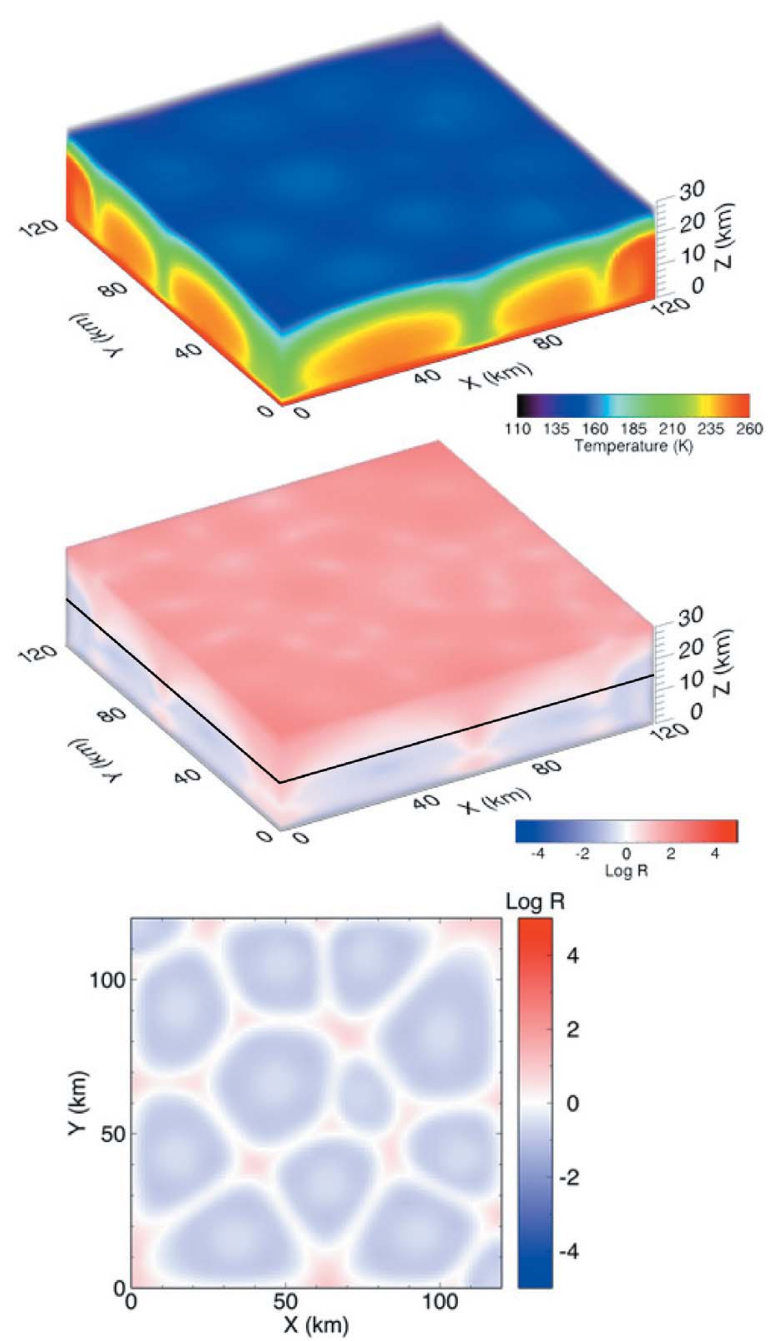

Figure 3. (top) Temperature, (middle) $R$, and (bottom) slice of $R$ values at the depth where the horizontally averaged $T=240 \mathrm{~K}$ isotherm for a $120 \times 120 \times 30 \mathrm{~km}$ section of the ice I shell of a Galilean satellite with $d=0.3 \mathrm{~mm}$ $\left(R a_{o}=1.4 \times 10^{6}\right)$. In Figures 3 (middle) and 3 (bottom), red areas within convective downwellings are locations where COF is created. Blue areas show locations where strain is accommodated by volume diffusion, which destroys COF.

[Greeley et al., 2004]. Past convection could be detected by looking for a layer of relict crystal fabric within a conductive ice shell. The presence of COF beneath surface features such as europan bands or Ganymedean grooved terrain could test the hypotheses that these features formed from extensional strain associated with upwellings of warm ice. For example, gray bands on Europa have been suggested to form in a manner similar to terrestrial midocean ridges [Prockter et al., 2002]. Modeling of their formation suggests $\varepsilon \gg 1$, extensional strain accommodated by dislocation creep (i.e., $R \gg 1$ ), and $T \sim 260 \mathrm{~K}$ only a few kilometers below the surface [Stempel et al., 2005]. COF could also form beneath Ganymede's grooved terrain, where $\varepsilon \sim 0.5$ to 2 [Pappalardo and Collins, 2005], flow in the subsurface is accommodated by dislocation creep, and $T>240 \mathrm{~K}$ at a depth of a few kilometers [Dombard and McKinnon, 2001].
[18] Acknowledgments. This work is supported by the NASA OPR grant NNX09AP30G to A. C. Barr and NASA PG\&G grant NNX10AJ65G to D. E. Stillman. We thank Roger Phillips and Joe MacGregor for helpful discussions.

[19] The Editor would like to thank the two anonymous reviewers for their assistance in evaluating this paper.

\section{References}

Alley, R. (1988), Fabrics in polar ice sheets: Development and prediction, Science, 240, 493-495.

Barr, A. C., and W. B. McKinnon (2007), Convection in ice I shells and mantles with self-consistent grain size, J. Geophys. Res., 112, E02012, doi:10.1029/2006JE002781.

Barr, A. C., and A. P. Showman (2009), Heat transfer in Europa's icy shell, in Europa, edited by Pappalardo, R. T., W. B. McKinnon, and K. K. Khurana, pp. 405-430, Univ. of Ariz. Press, Tucson.

Blankenship, D. D. (1989), Seismological investigations of a West Antarctic ice stream, Ph.D. thesis, Univ. of Wisc.-Madison, Madison.

Blankenship, D. D., D. A. Young, W. B. Moore, and J. C. Moore (2009), Radar sounding of Europa's subsurface properties and processes: The view from Earth, in Europa, edited by R. T. Pappalardo, W. B. McKinnon, and K. K. Khurana, pp. 631-654, Univ. of Ariz. Press, Tucson.

Budd, W. F., and T. H. Jacka (1989), A review of ice sheet rheology for ice sheet modeling, Cold Reg. Sci. Technol., 16, 107-144.

Chyba, C. F., S. J. Ostro, and B. C. Edwards (1998), Radar detectability of a subsurface ocean on Europa, Icarus, 134, 292-302.

Cuffey, K. M., and W. S. B. Paterson (2010), The Physics of Glaciers, 4th ed., Elsevier, New York.

Dall, J. (2009), Polarimetric ice sounding at P-band: First results, in IEEE 2009 International Geoscience and Remote Sensing Symposium, pp. 1024-1027, Inst. of Electr. and Electron. Eng., Piscataway, N. J.

Dall, J. (2010), Ice sheet anisotropy measured with polarimetric ice sounding radar, in IEEE 2010 International Geoscience and Remote Sensing Symposium, pp. 2507-2510, Inst. of Electr. and Electron. Eng., Piscataway, N. J.

Dombard, A. J., and W. B. McKinnon (2001), Formation of grooved terrain on Ganymede: Extensional instability mediated by cold, superplastic creep, Icarus, 154, 321-336.

Durand, G., et al. (2006), Effect of impurities on grain growth in cold ice sheets, J. Geophys. Res., 111, F01015, doi:10.1029/2005JF000320.

Evans, S. (1965), Dielectric properties of ice and snow-A review, J. Glaciol., 5, 773-792.

Freeman, J., L. Moresi, and D. A. May (2006), Thermal convection with a water ice I rheology: Implications for icy satellite evolution, Icarus, 180 , 251-264, doi:10.1016/j.icarus.2005.07.014.

Fujita, S., S. Mae, and T. Matsuoka (1993), Dielectric anisotropy in ice Ih at $9.7 \mathrm{GHz}$, Annal. Glaciol., 17, 276-280.

Glen, J. W., and J. G. Paren (1975), The electrical properties of snow and ice, J. Glaciol., 15, 15-38.

Goldsby, D. L., and D. L. Kohlstedt (2001), Superplastic deformation of ice: Experimental observations, J. Geophys. Res., 106, 11,017-11,030.

Goldsby, D. L., and D. L. Kohlstedt (2002), Reply to comment by P. Duval and M. Montagnat on "Superplastic deformation of ice: Experimental observations", J. Geophys. Res., 107(B11), 2313, doi:10.1029/ $2002 J B 001842$

Goodman, D. J., H. J. Frost, and M. F. Ashby (1981), The plasticity of polycrystalline ice, Philos. Mag. A, 43, 665-695.

Greeley, R. C., C. Chyba, J. W. Head, T. McCord, W. B. McKinnon, and R. T. Pappalardo (2004), Geology of Europa, in Jupiter: The Planet, Satellites and Magnetosphere, pp. 329-362, Cambridge Univ. Press, New York.

Grimm, R. E., D. E. Stillman, S. F. Dec, and M. A. Bullock (2008), Lowfrequency electrical properties of polycrystalline saline ice and salt hydrates, J. Phys. Chem. B, 112, 15,382-15,390.

Han, L., and A. P. Showman (2010), Coupled convection and tidal dissipation in Europa's ice shell, Icarus, 207, 834-844.

Hargreaves, N. D. (1977), The polarization of radio signals in the radio echo sounding of ice sheets, J. Appl. Phys. D, 10, 1285-1304.

Herrick, D. L., and D. J. Stevenson (1990), Extensional and compressional instabilities in icy satellite lithospheres, Icarus, 85, 191-204, doi:10. 1016/0019-1035(90)90110-U.

Johari, G. P., and P. A. Charette (1975), The permittivity and attenuation in polycrystalline ice and single-crystal ice Ih at 35 and $60 \mathrm{MHz}, J$. Glaciol., $14,293-303$.

Karato, S.-I. (2008), Deformation of Earth Materials, Cambridge Univ. Press, New York.

Kirk, R. L., and D. J. Stevenson (1987), Thermal evolution of a differentiated Ganymede and implications for surface features, Icarus, 69, 91-134. 
Matsuoka, K., T. Furukawa, S. Fujita, H. Maeno, S. Uratsuka, R. Naruse, and O. Watanabe (2003), Crystal orientation fabrics within the Antarctic ice sheet revealed by a multipolarization plane and dual-frequency radar survey, J. Geophys. Res., 108(B10), 2499, doi:10.1029/2003JB002425.

McNamara, A. K., S.-I. Karato, and P. E. van Keken (2001), Localization of dislocation creep in the lower mantle: Implications for the origin of seismic anisotropy, Earth Planet. Sci. Lett., 191, 85-99.

Mitri, G., and A. P. Showman (2008), A model for the temperaturedependence of tidal dissipation in convective plumes in icy satellites: Implications for Europa and Enceladus, Icarus, 195, 758-764.

Moore, J. C. (2000), Models of radar absorption in Europan ice, Icarus, $147,292-300$

Moresi, L.-N., and V. S. Solomatov (1995), Numerical investigation of 2D convection with extremely large viscosity variations, Phys. Fluids, 7 , 2154-2162.

Nimmo, F., and E. Gaidos (2002), Strike-slip motion and double ridge formation on Europa, J. Geophys. Res., 107(E4), 5021, doi:10.1029/ 2000JE001476.

Ojakangas, G. W., and D. J. Stevenson (1989), Thermal state of an ice shell on Europa, Icarus, 81, 220-241.

Pappalardo, R. T., and G. W. Collins (2005), Strained craters on Ganymede, J. Struct. Geol., 27, 827-838.

Pappalardo, R. T., G. C. Collins, J. W. Head, P. Helfenstein, T. McCord, J. M. Moore, L. M. Prockter, P. M. Schenk, and J. R. Spencer (2004) Geology of Ganymede, in Jupiter: The Planet, Satellites and Magnetosphere, pp. 363-396, Cambridge Univ. Press, New York.

Prockter, L. M., J. W. Head III, R. T. Pappalardo, R. J. Sullivan, A. E. Clifton, B. Giese, R. Wagner, and G. Neukum (2002), Morphology of Europan bands at high resolution: A mid-ocean ridge-type rift mechanism, J. Geophys. Res., 107(E5), 5028, doi:10.1029/2000JE001458.

Siegert, M., and S. Fujita (2001), Internal ice-sheet radar layer profiles and their relation to reflection mechanisms between Dome $\mathrm{C}$ and the Transantarctic mountains, J. Glaciol., 47, 205-212.

Siegert, M., and R. Kwok (2000), Ice-sheet radar layering and the development of preferred crystal orientation fabrics between Lake Vostok and Ridge B, central East Antarctica, Earth Planet. Sci. Lett., 179, 227-235.

Solomatov, V. S. (1995), Scaling of temperature- and stress-dependent viscosity convection, Phys. Fluids, 7, 266-274.

Solomatov, V. S., and A. C. Barr (2007), Onset of convection in fluids with strongly temperature-dependent, power-law viscosity: 2 . Dependence on the initial perturbation, Phys. Earth Planet. Inter., 165, 1-13.

Solomatov, V. S., and L.-N. Moresi (2000), Scaling of time-dependent stagnant lid convection: Application to small-scale convection on Earth and other terrestrial planets, J. Geophys. Res., 105, 21,795-21,818.

Stempel, M. M., A. C. Barr, and R. T. Pappalardo (2005), Model constraints on the opening rates of bands on Europa, Icarus, 177, 297-304

Tobie, G., G. Choblet, and C. Sotin (2003), Tidally heated convection: Constraints on Europa's ice shell thickness, J. Geophys. Res., 108 (E11), 5124, doi:10.1029/2003JE002099.

van den Berg, A. P., P. E. van Keken, and D. A. Yuen (1993), The effects of a composite non-Newtonian and Newtonian rheology on mantle convection, Geophys. J. Int., 115, 62-78.

A. C. Barr and D. E. Stillman, Department of Space Studies, Southwest Research Institute, 1050 Walnut St., Ste. 300, Boulder, CO 80302, USA. (amy@boulder.swri.edu) 